\title{
Queue-Aware Optimal Resource Allocation for the LTE Downlink
}

\author{
Hussam Ahmed, Krishna Jagannathan, Srikrishna Bhashyam \\ Department of Electrical Engineering \\ IIT Madras, Chennai, India
}

\begin{abstract}
We address the problem of optimal downlink resource allocation in an OFDMA system, in a scenario where very limited channel quality information (CQI) is available at the basestation. Our work is particularly applicable in the context of the LTE downlink, since the feedback mechanism we consider closely resembles one of the CQI reporting modes in LTE. Specifically, the users only report the indices of their best $M$ sub-bands and an effective CQI corresponding to these best $M$ bands. Our policy simultaneously performs optimal sub-band assignment and rate allocation, by taking into account channel quality as well as the queue backlogs of each user. The technical novelty of our work lies in exploiting a limit theorem on the best SNRs reported by the users, and combining it within a Lyapunov stability framework. We show that our policy is throughput maximizing among all policies which are constrained to the CQI mechanism considered. Numerical results indicate that in terms of throughput and average delay, our policy compares favorably to existing resource allocation policies such as proportional fair.
\end{abstract}

\section{INTRODUCTION}

Orthogonal Frequency Division Multiplexing (OFDM) is employed in most of the emerging high data-rate wireless cellular standards such as Long Term Evolution (LTE) [1] and IEEE 802.16 (WiMAX). In this paper, we tackle the problem of optimal resource allocation in downlink of an OFDMA system, in a scenario where very limited channel quality information (CQI) is available at the base-station (BS). Our work is particularly applicable in the context of LTE downlink, since the feedback mechanism we consider closely resembles one of the CQI reporting modes in LTE.

In an OFDM system such as the LTE downlink, the available bandwidth (say $20 \mathrm{MHz}$ ) is divided into several hundred subcarriers (e.g., 512, 1024, or 2048). These sub-carriers need to be allocated to multiple user equipments (UEs). In practice, a resource block (RB) pair consisting of 12 contiguous subcarriers and 14 OFDM symbols in time is the smallest resource allocation unit [2]. After accounting for unusable tones, this leaves us with about 50 to $100 \mathrm{RBs}$ to allocate to the UEs.

In order to schedule the UEs opportunistically, the basestation, in principle, needs to obtain channel quality information from each UE, on each of the resource blocks. This is highly impractical, since it leads to an enormous amount of control overheads on the uplink. To overcome this, UEs in an LTE system report CQI to the base-station in a very sparse manner.

\section{A. Related Work}

Various reduced feedback mechanisms have been studied in the literature in the context of resource allocation in OFDM downlink. In [3], the CQI of each UE is fed back only for those sub-bands ${ }^{1}$ whose quality is better than a certain threshold. The feedback overhead is even more reduced in [4], where the UEs report one-bit per sub-band whenever the channel quality exceeds the threshold. In [5], an opportunistic feedback strategy is considered wherein only the channel gains of prespecified number of best sub-bands are reported. A variation of this policy has been considered in [6], [7]. In [6], the UEs feedback the average gain of the best $M$ sub-bands and the corresponding indices while in [7], each UE reports an Effective Exponential Signal-to-noise ratio Mapping (EESM) of the best $M$ sub-bands and their respective indices. In effect, EESM translates the different SNRs on parallel channels into a single effective flat-fading SNR [8].

In this paper, we assume a CQI feedback mechanism similar to [6], [7], since it closely resembles one of the CQI reporting modes - namely, the UE-selected sub-band feedback mode defined in the LTE standards [9]. Specifically, the UEs only report the indices of their best $M$ sub-bands, where $M$ is a small number (say 2 to 5), and the EESM corresponding to these best $M$ bands.

Downlink resource allocation for OFDM systems has been studied from various perspectives in recent years. In [10], resource allocation in downlink OFDM is posed as a utility maximization problem, which includes proportionally fair resource allocation [11], [12] as a special case. The optimal power and sub-carrier allocation are then determined using convex duality techniques. While [10] assumed full CQI availability except for an estimation error term, [13] takes imperfect CQI into account by factoring for outages due to erroneous CQI at the base-station. In [14], the authors consider opportunistic resource allocation in OFDM under various fairness constraints, and propose a Hungarian algorithm based solution. It is worth noting that [10], [13], [14] assume fully backlogged buffers (i.e., that the base-station always has data to send to the UEs), and do not consider any queuing dynamics.

There is a vast literature on optimal server allocation to constrained queueing systems with time-varying connectivities. Most of the literature in this area based on the landmark papers [15], [16] which introduced Lyapunov techniques for

\footnotetext{
${ }^{1}$ A sub-band typically consists of one to three resource blocks.
} 
resource allocation. Subsequently, these Lyapunov methods, which explicitly take queue lengths into account for making resource allocation decisions, have been applied in various contexts including high-speed switches [17], satellites [18], wireless [19], and optical networks [20]. In addition to being inherently throughput maximizing, Lyapunov based resource allocation policies can also been used to ensure Quality of Service (QoS) metrics such as delay guarantees [21], [22] and fairness [23].

In the above Lyapunov based resource allocation policies, the resource allocation decision is based on the UE's channel quality as well as queue backlogs, and these are typically assumed to be available perfectly and instantaneously at the base station. In contrast, [24] proposes a throughput optimal resource allocation algorithm under delayed channel information; their policy utilizes the conditional expectation of the channel quality, given the delayed measurements. In [25], a cross layer resource allocation policy which maximizes the throughput under delayed CQI and takes into account the channel outage event is proposed. There has also been recent work on low-complexity dynamic resource allocation for OFDM [26], [27] to ensure low delay, but these papers do not consider sparse CQI feedback.

\section{B. Our Contributions}

In the present paper, we propose a queue-aware resource allocation policy for the OFDM downlink that is optimized for the specific form of the CQI available at the base-station. As described earlier, we assume that the UEs only report the indices of their best $M$ sub-bands, where $M$ is a small number, and the EESM corresponding to these best $M$ subbands. We develop a sub-band assignment and rate allocation algorithm which is throughput maximizing under this CQI scenario, when the total number of sub-bands is large. In other words, our algorithm is guaranteed to keep the queueing system stable for all traffic rates that can be stabilized by any resource allocation policy which is constrained to this CQI scenario.

One of the technical contributions of the paper lies in obtaining an explicit characterization of the outage probability on each of the $M$ reported sub-bands. In order to obtain the outage probability expression, we exploit a 'Gumbel' limit theorem on the joint distribution of the best $M$ sub-bands, which subsequently leads to an explicit expression for the conditional density, given the EESM. It is worth commenting that the Gumbel weak limit is an attractor for the extremal values of a fairly large family of distributions [28], so that our work does not crucially depend on the assumption that the sub-band gains are i.i.d. Rayleigh distributed. Another distinguishing feature of our resource allocation policy is that it naturally decouples for each sub-band, and does not entail solving any computationally intensive matching problems [14], [26].

\section{SySTEM MOdEL}

Consider a downlink system with one BS and $K$ UEs. The BS maintains a separate queue corresponding to each UE. Time is slotted, and the queue corresponding to $i^{\text {th }} \mathrm{UE}$ receives exogenous arrivals according to a random process. We denote the amount of data that enters queue $i$ during time slot $t$ by $A_{i}(t)$, and the queue length corresponding to the $i^{t h}$ UE during slot $t$ by $Q_{i}(t)$. We assume that the arrival process $A_{i}(t)$ is i.i.d. from slot to slot, with mean $\lambda_{i}$ and a finite second moment.

We assume that the channel between the BS and $i^{t h} \mathrm{UE}$ is a frequency selective Rayleigh fading channel. We remark that this Rayleigh fading assumption is not crucial to our work, but it makes exposition easier. OFDM transmission with $N_{c}$ sub-carriers is used. The SNR for the $i^{t h} \mathrm{UE}$ on the $j^{\text {th }}$ subcarrier follows an exponential distribution. The average SNR for the $i^{t h} \mathrm{UE}$ is denoted as $\gamma_{a v e, i}$.

We assume that the downlink channel gains of the UEs are not known to the BS unless the UEs feedback their CQI to the BS. This corresponds to a scenario where the uplink and the downlink channels are not reciprocal, or a scenario where the UEs are not transmitting any data on the uplink, so that reciprocity (even if present) cannot be exploited. In order to reduce feedback overhead, we assume that the sub-carriers are grouped into $N$ sub-bands in such a way that the channel can be approximated as flat-fading in each sub-band. Further, we consider the 'best $M$ ' feedback mechanism similar to [7], where each UE reports (i) the EESM corresponding to its best $M(\ll N)$ sub-bands, and (ii) the indices of those sub-bands.

Let $\gamma_{i}^{j}(t)$ be the SNR on the $j^{t h}$ sub-band for the $i^{t h} \mathrm{UE}$ in slot $t$ and $\gamma_{i}^{(1)}(t), \gamma_{i}^{(2)}(t) \ldots \gamma_{i}^{(N)}(t)$ be the ordered sub-band SNRs for $i^{t h}$ UE in descending order. The EESM for the best $M$ sub-bands corresponding to the $i^{\text {th }} \mathrm{UE}$ in slot $t$, denoted $\gamma_{i}^{e f f}$, is defined by

$$
\gamma_{i}^{e f f}(t)=-\eta \ln \left(\frac{1}{M} \sum_{j=1}^{M} e^{-\frac{\gamma_{i}^{(j)}(t)}{\eta}}\right),
$$

where $\eta$ is a parameter that depends on the modulation and coding scheme (MCS). Since we are considering a downlink problem, the BS is assumed to know the instantaneous queue lengths $Q_{i}(t)$ for all the UEs.

\section{Problem Formulation}

In this section, we develop a mathematical formulation of the optimal resource allocation problem. As mentioned earlier, the following information is assumed to be available with the BS during time-slot $t$ (for simplified notation, we omit $t$ ):

(i) The EESMs $\underline{\gamma}^{\text {eff }}=\left[\gamma_{1}^{\text {eff }}, \gamma_{2}^{\text {eff }}, \ldots, \gamma_{K}^{\text {eff }}\right]$

(ii) The index sets $\underline{I}=\left[I_{1}, I_{2}, \ldots, I_{K}\right]$

(iii) The queue length vector $\underline{Q}=\left[Q_{1}, Q_{2}, \ldots, Q_{K}\right]$.

where $I_{i}$ is the set of indices reported by the $i^{\text {th }} \mathrm{UE}$ and is given by $I_{i}=\left\{i_{1}, i_{2}, \ldots i_{M}\right\}$ where $i_{j}$ is the index of the $j^{\text {th }}$ best sub-band of the $i^{\text {th }}$ UE. Given this information, our aim is to come up with a resource allocation policy which 
can maximize throughput while keeping all queues at the BS stable. In order to make this statement precise, we develop some terminology and notation.

A resource allocation policy performs the following two operations in each slot.

- Sub-band assignment: For each sub-band $j$ that is reported by at least one UE, the policy determines a unique UE to assign the sub-band. (Recall that a sub-band can be allocated to at most one UE due to interference considerations, whereas a UE can be allocated multiple sub-bands).

- Rate allocation: Given that $j^{\text {th }}$ sub-band is assigned to $i^{t h} \mathrm{UE}$, determine the rate $r_{i, j}$ at which data transmission will take place on $j^{\text {th }}$ sub-band.

From now on, we use the notation $[i, j]$ for the $i^{\text {th }} \mathrm{UE}$ - $j^{\text {th }}$ sub-band pair. In the interest of simplicity, we restrict our attention to policies which allocate equal power to all scheduled sub-bands, although our framework can be modified to include optimal power allocation for different sub-bands. To be precise, define $\hat{I}=\cup_{i=1}^{K} I_{i}$ as the set of all distinct subbands reported by at least one UE, and let $M^{\prime}=|\hat{I}|$ denote the number of such distinct sub-bands. Assume that the BS has a power budget of $P$ for transmissions during each slot. Then, the base station allocates power $P / M^{\prime}$ to each sub-band. Let $C_{i, j}$ be the instantaneous capacity of $[i, j]$. Under the above assumptions, we have

$$
C_{i, j}=\log _{2}\left(1+\frac{P}{M^{\prime}} \gamma_{i}^{j}\right) .
$$

For a reliable communication over a sub-band, the rate assigned to $[i, j], r_{i, j}$, should not exceed $C_{i, j}$. Given $\underline{\gamma}^{\text {eff }}$ and the index sets $\underline{I}$, we say $[i, j]$ is in outage if the rate allocated to $[i, j]$ is greater than $C_{i, j}$. The outage probability for $[i, j]$ when the assigned rate is $r_{i, j}$ is defined as follows:

$$
P_{i, j}\left(r_{i, j}\right)=\mathbb{P}\left\{C_{i, j}<r_{i, j} \mid \gamma_{i}^{e f f}, I_{i}\right\} .
$$

We define a natural metric, namely goodput, as the average successfully transmitted rate over a sub-band [29]. The goodput for $[i, j]$ when the assigned rate is $r_{i, j}$ is defined as follows:

$$
G_{i, j}\left(r_{i, j}\right)=r_{i, j}\left(1-P_{i, j}\left(r_{i, j}\right)\right) .
$$

Next, we briefly review the queueing dynamics and stability considerations of the queueing system at the BS.

\section{A. Stability considerations}

The queue evolution equation for the $i^{t h} \mathrm{UE}$ can be written as

$$
Q_{i}(t+1)=\max \left\{Q_{i}(t)-\mu_{i}(t), 0\right\}+A_{i}(t),
$$

where $A_{i}(t)$ and $\mu_{i}(t)$ are arrival and service processes of the $i^{t h}$ UE queue. Here, $\mu_{i}(t)$ is the amount of data served from the $i^{t h} \mathrm{UE}$ queue during slot $t$, and can be written as

$$
\mu_{i}(t)=\sum_{j=1}^{N} a_{i, j} r_{i, j} H_{i, j}(t) .
$$

In the above expression, $a_{i, j}$ denotes the fraction of time the $j^{\text {th }}$ sub-band is allocated to the $i^{\text {th }}$ UE during slot $t$. Clearly

$$
\sum_{i=1}^{K} a_{i, j} \leq 1
$$

Later, we will show that our optimal policy allocates a subband to at most one UE during each time-slot. Next, $H_{i, j}(t)$ is an indicator random variable which takes a value 1 whenever the transmission through $[i, j]$ during slot $t$ is successful and 0 otherwise. Thus, $\mathbb{P}\left\{H_{i, j}(t)=0\right\}=P_{i, j}^{N}\left(r_{i, j}\right)$.

In the spirit of [30], we say that the queueing system at the $\mathrm{BS}$ is strongly stable if for each UE $i$,

$$
\limsup _{T \rightarrow \infty} \frac{1}{T} \sum_{t=0}^{T} E\left[Q_{i}(t)\right]<\infty .
$$

Denote by $\mathcal{P}$ the family of all resource allocation which allocate equal power to all scheduled sub-bands, and have access only to the parameters $\underline{\gamma}^{e f f}, \underline{I}$, and $\underline{Q}$ in order to make the resource allocation decisions during each slot. Let $\Lambda$ be the stability region of the network, which is defined as (the closure of) the set of all arrival rates $\underline{\lambda}=\left(\lambda_{1}, \lambda_{2}, \ldots, \lambda_{K}\right)$ for which there exists some policy $\Pi \in \mathcal{P}$ under which the queueing system is strongly stable.

We find a resource allocation policy in $\mathcal{P}$ which is throughput optimal, in the sense that it keeps the queuing system stable for all arrival rates in the interior of $\Lambda$.

\section{Throughput Optimal Resource Allocation POLICY}

During each time slot, the scheduler at the BS observes $\underline{\gamma}^{e f f}, \underline{I}$, and $\underline{Q}$, and implements the following steps :

1) Determine $\hat{I}=\cup_{i=1}^{K} I_{i}$ and $M^{\prime}=|\hat{I}|$.

2) for $j=1$ to $M^{\prime}$ do

(i) Set $P_{j}=\frac{P}{M^{\prime}}$.

(ii) Determine $U_{j}=\left\{i \mid j \in I_{i}\right\}$.

(iii) Calculate an estimate of the outage probability $\hat{P}_{i, j}(r)$ as a function of $r$ for each $i \in U_{j}$. (See Section V)

(iv) Calculate

$$
r_{i, j}^{*}=\arg \max _{r}\left\{r\left(1-\hat{P}_{i, j}(r)\right)\right\} \forall i \in U_{j} .
$$

(v) Calculate

$$
i(j)=\arg \max _{i \in U_{j}}\left\{Q_{i}(t) r_{i, j}^{*}\left(1-\hat{P}_{i, j}\left(r_{i, j}^{*}\right)\right)\right\} .
$$

(vi) Assign $j^{\text {th }}$ sub-band to $i(j)^{t h} \mathrm{UE}$, and transmit at rate $r_{i(j), j}^{*}$ with power $P_{j}$.

3) end for

\section{A. Discussion}

In the first step, the scheduler determines the set of all distinct sub-bands reported by the UEs. Then, for each such sub-band $j$, the scheduler sets the power to be $P_{j}$ and determines the set $U_{j}$ of all UEs who report that sub-band as being one of their best $M$ sub-bands. In the next step, 
the outage probability on $[i, j]$ is computed, as explained in Section V. Then the scheduler computes the rate that ensures the best goodput for each UE $i \in U_{j}$. Finally, in the last two steps, the scheduler assigns $j^{t h}$ sub-band to the $i^{\text {th }} \mathrm{UE}$ that has the maximum queue-length goodput product.

Notice that the above algorithm assigns every reported subband to a unique UE. Also, no power is assigned to sub-bands that are not reported by any UE.

\section{B. Lyapunov Analysis}

In this section, we derive the optimal resource allocation policy as a Lyapunov drift minimizing policy, and prove that it is throughput optimal. We define the quadratic Lyapunov function

$$
L(\underline{Q}(t))=\sum_{i=1}^{K}\left(Q_{i}(t)\right)^{2},
$$

and consider the conditional Lyapunov drift

$$
\Delta(\underline{Q}(t))=\mathbb{E}\{L(\underline{Q}(t+1))-L(\underline{Q}(t)) \mid \underline{Q}(t)\} .
$$

Using (5), straightforward algebra gives

$$
\begin{gathered}
L(\underline{Q}(t+1))-L(\underline{Q}(t)) \leq \sum_{i=1}^{K}\left(A_{i}(t)\right)^{2}+2 \sum_{i=1}^{K} A_{i}(t) Q_{i}(t) \\
\left(\sum_{j=1}^{N} \sum_{i=1}^{K} a_{i, j} r_{i, j} H_{i, j}(t)\right)^{2}-2 \sum_{i=1}^{K} Q_{i}(t) \sum_{j=1}^{N} a_{i, j} r_{i, j} H_{i, j}(t) .
\end{gathered}
$$

Thus, taking conditional expectations and exploiting the independence of $A_{i}(t)$ and $Q_{i}(t)$, we get

$$
\begin{aligned}
\Delta(\underline{Q}(t)) \leq B & +2 \sum_{i=1}^{K} Q_{i}(t) \lambda_{i} \\
& -2 \sum_{i=1}^{K} \sum_{j=1}^{N} Q_{i}(t) a_{i, j} r_{i, j}\left(1-P_{i, j}\left(r_{i, j}\right)\right),
\end{aligned}
$$

where

$$
B=\left(\sum_{j=1}^{N} \max _{i}\left\{C_{i, j}\right\}\right)^{2}+\sum_{i=1}^{K} \mathbb{E}\left[A_{i}(t)^{2}\right]<\infty .
$$

We know from [30, Lemma 4.1] that the Lyapunov drift becoming negative for large queue backlogs is a sufficient condition for the strong stability of the queueing system. With this in mind, we seek the policy that maximizes the negative term on the right hand side of (9). We therefore formulate the optimal resource allocation problem as follows.

$$
\max _{\left\{a_{i, j}\right\},\left\{r_{i, j}\right\}} \sum_{i=1}^{K} \sum_{j=1}^{N} Q_{i}(t) a_{i, j} G_{i, j}\left(r_{i, j}\right),
$$

subject to

$$
\begin{aligned}
\sum_{i=1}^{K} a_{i, j} & \leq 1, \forall j, \\
a_{i, j} & \geq 0, \forall i, j \\
r_{i, j} & \geq 0, \forall i, j .
\end{aligned}
$$

We assume that it is possible to come up with modulation and coding schemes for any desired rate $r_{i, j}$. Then, the above problem is a convex optimization problem and the solution can be obtained easily by using Karush-Kuhn-Tucker (KKT) conditions [31]. The solution is discussed next.

\section{Minimizing the Lyapunov Drift}

We now solve the convex optimization problem (10) and arrive at our resource allocation policy. Introducing the nonnegative Lagrange multipliers $\left\{\alpha_{j}\right\},\left\{\beta_{i, j}\right\},\left\{\delta_{i, j}\right\}$ for constraints $(C 1)-(C 3)$ respectively, the following conditions also must be satisfied at the optimal solution (superscript $(\cdot)^{*}$ denotes optimal values).

$$
\begin{aligned}
Q_{i}(t) G_{i, j}\left(r_{i, j}^{*}\right)+\beta_{i, j}^{*}-\alpha_{j}^{*}=0, \forall i, j . \\
Q_{i}(t) a_{i, j}^{*} \frac{\partial G_{i, j}\left(r_{i, j}^{*}\right)}{\partial r_{i, j}^{*}}+\delta_{i, j}^{*}=0, \forall i, j . \\
\alpha_{j}^{*}\left(\sum_{i=1}^{K} a_{i, j}^{*}-1\right)=0, \forall j . \\
\beta_{i, j}^{*} a_{i, j}^{*}=0, \forall i, j . \\
\delta_{i, j}^{*} r_{i, j}^{*}=0, \forall i, j .
\end{aligned}
$$

Proposition 1. The optimal sub-band allocation for problem (10) assigns a sub-band exclusively to the UE with the largest corresponding queue-length goodput product.

Proof. It follows from (15) and (12) that if $r_{i, j}^{*}>0$, then $\delta_{i, j}^{*}=0$, i.e., $\frac{\partial G_{i, j}\left(r_{i, j}^{*}\right)}{\partial r_{i, j}^{*}}=0$. Thus, $r_{i, j}^{*}$ is obtained by maximizing goodput of $i^{\text {th }} \mathrm{UE}$ on $j^{\text {th }}$ sub-band (Step 5 of our policy in Section IV). Similarly, it follows from (14) and (11) that if $a_{i, j}^{*}>0$, then $\beta_{i, j}^{*}=0$, i.e., $Q_{i}(t) G_{i, j}\left(r_{i, j}^{*}\right)=\alpha_{j}^{*}$. If $a_{i, j}^{*}=0$, then $\beta_{i, j}^{*} \geq 0$, i.e., $Q_{i}(t) G_{i, j}\left(r_{i, j}^{*}\right) \leq \alpha_{j}^{*}$. Hence, the $j^{\text {th }}$ sub-band is assigned to the UE with largest queuelength goodput product $Q_{i}(t) G_{i, j}\left(r_{i, j}^{*}\right)$. If multiple UEs have the same queue-length goodput product for the same subband $j$, the sub-band can be shared in any arbitrary manner among these users without affecting optimality in terms of the objective function in (10).

Proposition 1 shows that the optimal sub-band allocation assigns each reported sub-band $j$ to the UE which has the maximum queue-length goodput product on the $j^{t h}$ sub-band. This establishes that the policy in Section IV minimizes the Lyapunov drift.

Since the proposed policy ensures the "most negative" Lyapunov drift among the class $\mathcal{P}$, it seems plausible that our policy should be able to stabilize the queueing system, whenever some policy in $\mathcal{P}$ can do so. The following theorem asserts that this is indeed true.

Theorem 1. The resource allocation policy proposed in Section IV is asymptotically throughput optimal, when the number of sub-bands is large.

The proof is relegated to Appendix A. Our policy is only asymptotically throughput optimal since the policy uses the 
limiting outage probability given by a limit theorem, instead of the actual outage probability which is difficult to compute.

\section{Derivation of Outage Probability}

In this section, we describe how the BS estimates the outage probability on $[i, j]$ in Step 4 of our algorithm, using only the parameters $\underline{\gamma}^{\text {eff }}$ and $\underline{I}$. We utilize a limit theorem on the order statistics of the SNRs to derive an expression for the conditional joint distribution of the SNRs on the best $M$ sub-bands for each UE, given the EESM and the subband indices. For ease of exposition, we assume that the SNRs on the sub-bands of a given UE are i.i.d. exponentially distributed. This assumption will hold well in the case of Rayleigh fading in a rich multi-path environment, with number of paths comparable to the number of sub-bands. However, we remark that the limit theorem we are about to exploit holds for a fairly large class of distributions - namely, those which lie within the Gumbel domain of attraction [28]. Therefore, our policy remains asymptotically throughput optimal for this class of sub-band SNR distributions.

We first state a result which follows from [32, Theorem 15] regarding the order statistics of $M$ extremal values, drawn from $N$ i.i.d. exponential random variables .

Theorem 2. Let $Z_{1}, Z_{2}, \ldots, Z_{N}$ be a sequence of i.i.d. unit exponential random variables, and $Z_{(1)}, Z_{(2)}, \ldots, Z_{(N)}$ be the corresponding order statistics in descending order. Then, for any finite $M$,

$$
\left(e^{-\tilde{Z}_{(1)}}, e^{-\tilde{Z}_{(2)}}, \ldots, e^{-\tilde{Z}_{(M)}}\right) \stackrel{\mathfrak{D}}{\longrightarrow}\left(Y_{1}, Y_{2}, \ldots Y_{M}\right),
$$

as $N \rightarrow \infty$, where $\tilde{Z}_{(i)}=Z_{(i)}-\ln N, Y_{i}=\sum_{j=1}^{i} X_{j}$ and $X_{j}$ s are i.i.d. unit exponential random variables.

Consider the $i^{\text {th }}$ UE. Let $S_{i}^{j}=e^{-\left(\frac{\gamma_{i}^{i_{j}}}{\gamma_{a v e, i}}-\ln N\right)}$ for $j=$ $1, \ldots, M$. Assuming that the number of sub-bands $N$ is large, we can use Theorem 2, to approximate the unconditional joint pdf of $\underline{S}_{i}^{(M)}=\left(S_{i}^{1}, S_{i}^{2}, \ldots, S_{i}^{M}\right)$ as

$f_{\underline{S}_{i}^{(M)}}\left(s_{1}, s_{2}, \ldots, s_{M}\right)=e^{-s_{M}}, \quad 0 \leq s_{1} \leq s_{2} \leq \ldots \leq s_{M}$.

Numerical results indicate that this approximation is good even for moderate values of $N$. Next, define $S_{i}^{\text {eff }}=$ $e^{-\left(\frac{\gamma_{i}^{e f f}}{\gamma_{a v e, i}}-\ln N\right)}$. Choosing the parameter $\eta$ in (1) as unity for simplicity, we have

$$
S_{i}^{e f f}=\frac{1}{M} \sum_{j=1}^{M} S_{i}^{j} .
$$

Note that $S_{i}^{e f f}$ is known to the BS. Next, conditioned on $S_{i}^{e f f}=s$ and $I_{i}=I, \underline{S}_{i}^{(M)}$ takes values only on the hyperplane $\frac{1}{M} \sum_{j=1}^{M} S_{i}^{j}=s$. Hence, we ignore the $M^{t h}$ best SNR and calculate the conditional joint pdfs for the remaining sub-band SNRs as follows.

$$
\begin{aligned}
& f_{\underline{S}_{i}^{(M-1)} \mid S_{i}^{e f f}=s, I_{i}=I}\left(s_{1}, s_{2}, \ldots, s_{M-1}\right) \\
& \quad=\frac{M e^{-\left(M s-\sum_{j=1}^{M-1} s_{j}\right)}}{f_{S_{i}^{e f f}}(s)}, 0 \leq s_{1} \leq s_{2} \leq \ldots \leq M s-\sum_{j=1}^{M-1} s_{j} .
\end{aligned}
$$

Now, we can find the conditional marginal density $f_{S^{j} \mid S_{i f}^{e f f}=s, I_{i}=I}\left(s_{j}\right)$ for $j=1, \ldots, M-1$ by integrating out the other variables in the above expression. The outage probability can thus be determined as

$$
\hat{P}_{i, i_{j}}(r)=\int_{y}^{N} f_{S_{i}^{j} \mid S_{i}^{e f f}=N e^{-\gamma, I_{i}=I}}\left(s_{j}\right) d s_{j} .
$$

where $y=N e^{-\left(\frac{2^{r}-1}{P \gamma_{a v e, i}}\right)}$. Similarly, $\hat{P}_{i, i_{M}}(r)$ can be computed from the conditional marginal of $S_{i}^{M}$. Closed form expressions for the outage probabilities (16) can be obtained through some tedious computations; in [33], we provide explicit expressions for the cases $M=3$ and $M=4$. The outage probabilities computed in (16) are used in Step 4 of the resource allocation algorithm.

\section{Simulation Results}

In this section, we present simulation results that demonstrate the throughput gains achieved by the proposed policy over other existing policies. We also demonstrate that the limiting approximation we use to obtain closed form outage probability expressions is a good approximation.

The proposed policy (labeled as "optimal" in the plots) is throughput optimal among all policies that use the limited channel feedback scheme described in Section II. Three important components of our policy are: (1) evaluation of the conditional expected CQI for each sub-band from the EESM, (2) evaluation of goodput while accounting for outage probability, and (3) optimal utilization of queue length information. To illustrate the importance of each component of our proposed policy, we compare the proposed policy with the following policies (each of the heuristic policies ignores at least one component of our proposed policy): (1) a throughput optimal policy with perfect CQI (labelled "Perfect CQI'), (2) a policy that uses queue length information but assumes that the reported EESM is the CQI for all the best $M$ reported sub-bands (labelled "Heuristic 1"), (3) a policy that uses queue length information and evaluates the conditional expected CQI for the best $M$ reported sub-bands without accounting for outage probability (labelled "Heuristic 2"), and (4) a proportionally fair rate allocation policy that uses the conditional expected CQI and goodput evaluation without using queue length information (labelled "PF").

A heterogeneous single-cell OFDM downlink with $K=$ 100 UEs at different distances from the BS is simulated. The $i^{\text {th }}$ UE is located at a distance of $i d_{0}$ from the BS where $d_{0}$ is a constant. The average SNR for a user is assumed to be inversely proportional to the square of its distance from the BS. The total number of subcarriers is 512 and there are 12 subcarriers in each sub-band. Two channel models are considered: (1) IID sub-bands, and (2) Correlated sub-bands resulting from a 6-path channel with an uniform power-delay profile where each path is Rayleigh fading. The arrival traffic for the $i^{t h} \mathrm{UE}$ is assumed to be Poisson with with parameter $\lambda_{i}$. The channel feedback from each UE is assumed to be the best $M$ sub-bands and EESM for these sub-bands. 
Figures 1 and 2 show the average queue length (averaged across UEs and time slots) versus the aggregate arrival rate (i.e., sum of $\lambda_{i}$ 's) for the IID and correlated sub-band cases respectively. $\lambda_{i}$ is chosen as $(K-i+1) \lambda$, i.e., each UE has a different arrival traffic rate, and $\lambda$ is varied to change the arrival traffic load. Also, $M=3$ and the number of sub-bands $N=$ 43. It is clear that the proposed policy can support significantly higher arrival traffic for the same average queue length than the heuristic policies. The Perfect CQI policy is also shown to quantify the loss due to limited feedback. It is also clear that the proposed policy provides similar performance gains even in the correlated sub-band case. Similar results have been observed for $M=4$ (not shown due to space limitations).

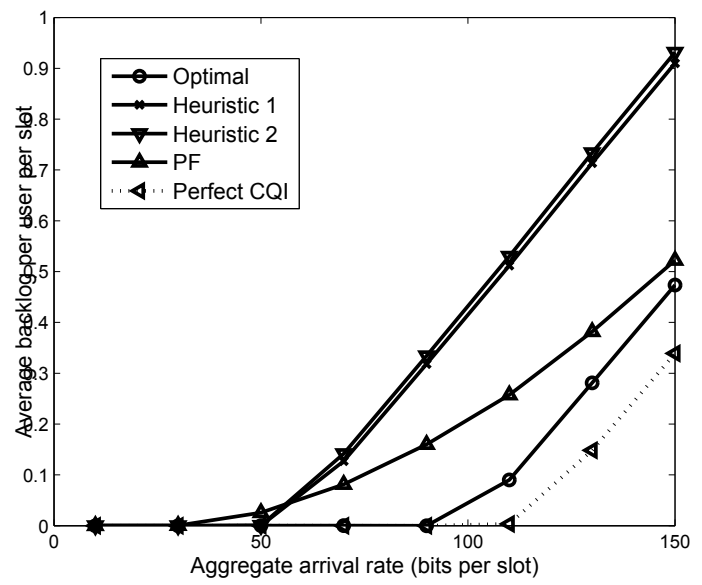

Fig. 1. IID sub-bands case: $M=3, N=43, \eta=1$.

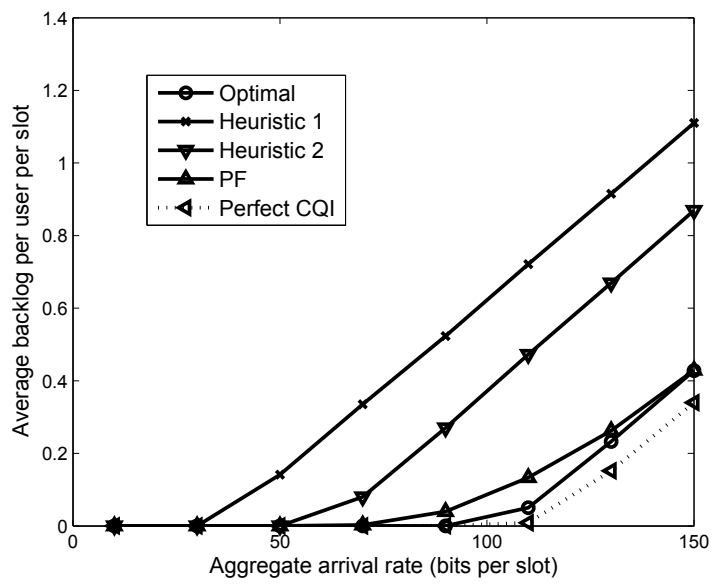

Fig. 2. Correlated sub-bands case: $M=3, N=43, \eta=1$.

Figure 3 shows the conditional CDF of the CQI of the best sub-band given a particular EESM for the best $M$ sub-bands. Four cases of $N$ (the total number of sub-bands) are shown. Note that the number of subcarriers is $12 N$. It can be observed that the weak limit approximation is very good for $N=22$ and $N=43$ for the IID case.
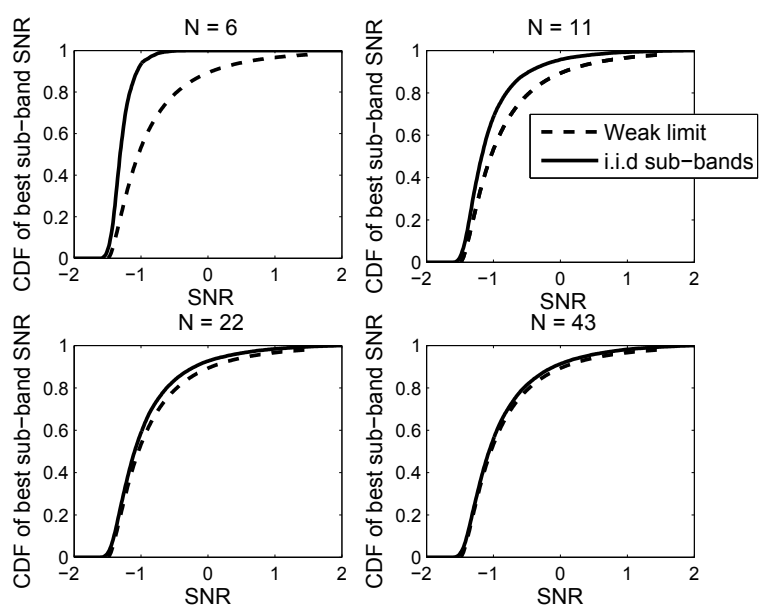

Fig. 3. Conditional CDF of best sub-band given $\gamma_{e f f}=-1.5+\log N$, $M=3, \eta=1$.

\section{CONCLUSION}

We proposed a queue-aware policy for allocating sub-bands in the LTE downlink when each UE reports the best $M$ subband indices and a single effective CQI for these bands. The throughput optimality of the proposed policy was shown using the Lyapunov stability framework. The policy assigns each sub-band to the UE with the best queue-length goodput product for that sub-band. The goodput was obtained by deriving analytical expressions for the conditional outage probability of each sub-band given the effective CQI. The conditional outage probability was derived by exploiting a limit theorem on the joint distribution of the SNR of the best sub-bands. The proposed policy supports signifcantly higher arrival traffic than existing policies like: (1) proportional fair allocation based on CQI that does not consider queue information, (2) queueaware policies that use the effective CQI as the CQI of each sub-band, and (3) queue-aware policies that do not account for outage in the estimation of goodput.

\section{APPENDIX A \\ PROOF OF THEOREM 1}

Proof. If the arrival rate vector $\underline{\lambda}$ is stabilizable by some policy $\Pi \in \mathcal{P}$ then $\exists \underline{\epsilon}=\left(\epsilon_{1}, \epsilon_{2}, \ldots, \epsilon_{K}\right)$ with $\epsilon_{i}>0 \forall i$ such that

$$
\lambda_{i} \leq \sum_{j=1}^{N} b_{i, j} r_{i, j}\left(1-P_{i, j}\left(r_{i, j}\right)\right)-\epsilon_{i}, \quad \forall i,
$$

where $b_{i, j}$ is the fraction of $j^{\text {th }}$ sub-band allocated to $i^{t h}$ UE and $r_{i, j}$ is the rate assigned to $[i, j]$ by policy $\Pi$. Next, we invoke Scheffé's Lemma [34], which asserts the uniform convergence of $\left|P_{i, j}(r)-\hat{P}_{i, j}(r)\right|$ to zero. Thus, for large $N$,

$$
\left|P_{i, j}(r)-\hat{P}_{i, j}(r)\right| \leq \delta_{i, j}^{N}, \forall r, i, j,
$$

where $\delta_{i, j}^{N}$ is a small positive number independent of $r$. Since for every sub-band, our policy assigns $a_{i, j}^{*}$ and $r_{i, j}^{*}$ such that 
$\sum_{i=1}^{K} Q_{i}(t) a_{i, j}^{*} r_{i, j}^{*}\left(1-\hat{P}_{i, j}\left(r_{i, j}^{*}\right)\right)$ is maximized, the following inequality holds good $\forall j,\left\{b_{i, j}\right\},\left\{r_{i, j}\right\}$.

$$
\begin{aligned}
\sum_{i=1}^{K} Q_{i}(t) b_{i, j} r_{i, j} & \left(1-P_{i, j}\left(r_{i, j}\right)-\delta_{i, j}^{N}\right) \leq \\
& \sum_{i=1}^{K} Q_{i}(t) a_{i, j}^{*} r_{i, j}^{*}\left(1-P_{i, j}\left(r_{i, j}^{*}\right)+\delta_{i, j}^{N}\right) .
\end{aligned}
$$

For our policy, the Lyapunov drift can be upper bounded as

$$
\Delta(\underline{Q}(t)) \leq B-\sum_{i=1}^{K} Q_{i}(t)\left(\epsilon_{i}-\sum_{j=1}^{N}\left(a_{i, j}^{*} r_{i, j}^{*}+b_{i, j} r_{i, j}\right) \delta_{i, j}^{N}\right) .
$$

Note that only $M$ of the $a_{i, j}^{*}$ and $b_{i, j}$ are non-zero for each user $i$. Thus, for any $\underline{\epsilon}$, there exists a large enough $N$ for which

$$
\epsilon_{i}-\sum_{j=1}^{N}\left(a_{i, j}^{*} r_{i, j}^{*}+b_{i, j} r_{i, j}\right) \delta_{i, j}^{N}>0, \forall i,
$$

which ensures that the Lyapunov drift becomes negative as queues grow. i.e., the proposed policy stabilizes all the arrival rates which can be stabilized by any other policy for large enough $N$. Hence it is asymptotically throughput optimal.

\section{ACKNOWLEDGMENT}

The authors thank Dr. Sheetal Kalyani (IIT Madras) for her useful comments. The second author acknowledges support from the IU-ATC project, funded by the Department of Science and Technology, Government of India, and the UK EPSRC Digital Economy programme.

\section{REFERENCES}

[1] C. Cox, An Introduction to LTE: LTE, LTE-Advanced, SAE and $4 G$ Mobile Communications. Wiley, 2012.

[2] E. Dahlman, S. Parkvall, J. Skold, and P. Beming, $3 G$ Evolution: HSPA and LTE for Mobile Broadband, 2nd ed. Academic Press, 2008.

[3] D. Gesbert and M. S. Alouini, "How much feedback is multi-user diversity really worth?" in Proc. IEEE ICC, vol. 4, 2004, pp. 234-238.

[4] S. Sanayei and A. Nosratinia, "Opportunistic downlink transmission with limited feedback," Information Theory, IEEE Transactions on, vol. 53, no. 11, pp. $4363-4372$, nov. 2007

[5] Y.-J. Choi and S. Bahk, "Selective channel feedback mechanisms for wireless multichannel scheduling," in Proceedings of the 2006 International Symposium on on World of Wireless, Mobile and Multimedia Networks. IEEE Computer Society, 2006, pp. 289-300.

[6] S. Donthi and N. Mehta, "An accurate model for eesm and its application to analysis of cqi feedback schemes and scheduling in lte," Wireless Communications, IEEE Transactions on, vol. 10, no. 10, pp. $3436-$ 3448, october 2011.

[7] _ - "Joint performance analysis of channel quality indicator feedback schemes and frequency-domain scheduling for lte," Vehicular Technology, IEEE Transactions on, vol. 60, no. 7, pp. 3096 -3109, sept. 2011.

[8] E. Westman, "Calibration and evaluation of the exponential effective sinr mapping (eesm) in 802.16," The Royal Institute of Technology (KTH) , Master's thesis, september 2006.

[9] "Evolved universal terrestrial radio access (E-UTRA); physical layer procedures (release 8)," TS 36.213 (v8.8.0), 3rd Generation Partnership Project (3GPP), Tech. Rep., 2009.

[10] J. Huang, V. G. Subramanian, R. Agrawal, and R. A. Berry, "Downlink scheduling and resource allocation for ofdm systems," Wireless Communications, IEEE Transactions on, vol. 8, no. 1, pp. 288-296, 2009.

[11] H. J. Kushner and P. A. Whiting, "Convergence of proportional-fair sharing algorithms under general conditions," Wireless Communications, IEEE Transactions on, vol. 3, no. 4, pp. 1250-1259, 2004.
[12] P. Viswanath, D. N. C. Tse, and R. Laroia, "Opportunistic beamforming using dumb antennas," Information Theory, IEEE Transactions on, vol. 48, no. 6, pp. 1277-1294, 2002.

[13] S. Stefanatos and N. Dimitriou, "Downlink ofdma resource allocation under partial channel state information," in Communications, 2009. ICC'09. IEEE International Conference on. IEEE, 2009, pp. 1-5.

[14] Z. Zhang, Y. He, and E. Chong, "Opportunistic downlink scheduling for multiuser ofdm systems," in Wireless Communications and Networking Conference, 2005 IEEE, vol. 2, march 2005, pp. 1206 - 1212 Vol. 2.

[15] L. Tassiulas and A. Ephremides, "Dynamic server allocation to parallel queues with randomly varying connectivity," Information Theory, IEEE Transactions on, vol. 39, no. 2, pp. $466-478$, mar 1993.

[16] _ "Stability properties of constrained queueing systems and scheduling policies for maximum throughput in multihop radio networks," Automatic Control, IEEE Transactions on, vol. 37, no. 12, pp. 1936 -1948 , dec 1992

[17] N. McKeown, A. Mekkittikul, V. Anantharam, and J. Walrand, "Achieving 100\% throughput in an input-queued switch," IEEE Transactions on Communications, vol. 47, no. 8, pp. 1260-1267, 1999.

[18] M. Neely, E. Modiano, and C. Rohrs, "Power and server allocation in a multi-beam satellite with time varying channels," in IEEE INFOCOM, vol. 3, 2002.

[19] — "Dynamic power allocation and routing for time varying wireless networks," in IEEE INFOCOM, vol. 1, 2003.

[20] A. Brzezinski and E. Modiano, "Dynamic reconfiguration and routing algorithms for IP-over-WDM networks with stochastic traffic," Journal of Lightwave Technology, vol. 23, no. 10, p. 3188, 2005.

[21] S. Kittipiyakul and T. Javidi, "Delay-optimal server allocation in multiqueue multiserver systems with time-varying connectivities," Information Theory, IEEE Transactions on, vol. 55, no. 5, pp. 2319 -2333, may 2009.

[22] J. J. Jaramillo, R. Srikant, and L. Ying, "Scheduling for optimal rate allocation in ad hoc networks with heterogeneous delay constraints," Selected Areas in Communications, IEEE Journal on, vol. 29, no. 5, pp. 979-987, 2011

[23] M. J. Neely, E. Modiano, and C.-P. Li, "Fairness and optimal stochastic control for heterogeneous networks," Networking, IEEE/ACM Transactions on, vol. 16, no. 2, pp. 396-409, 2008.

[24] L. Ying and S. Shakkottai, "On throughput optimality with delayed network-state information," Information Theory, IEEE Transactions on vol. 57, no. 8, pp. 5116-5132, 2011.

[25] C. Manikandan, S. Bhashyam, and R. Sundaresan, "Cross-layer scheduling with infrequent channel and queue measurements," Wireless Communications, IEEE Transactions on, vol. 8, no. 12, pp. $5737-5742$, december 2009.

[26] S. Bodas, S. Shakkottai, L. Ying, and R. Srikant, "Low-complexity scheduling algorithms for multi-channel downlink wireless networks," in INFOCOM, 2010, pp. 2222-2230.

[27] _ _ "Scheduling for small delay in multi-rate multi-channel wireless networks," in INFOCOM, 2011, pp. 1251-1259.

[28] E. Bertin and M. Clusel, "Generalised extreme value statistics and sum of correlated variables," Journal of Physics A, 2006

[29] S. Stefanatos and N. Dimitriou, "Downlink ofdma resource allocation under partial channel state information," in Communications, 2009. ICC '09. IEEE International Conference on, june 2009, pp. 1 -5.

[30] L. Georgiadis, M. Neely, and L. Tassiulas, Resource allocation and cross-layer control in wireless networks. Now Pub, 2006.

[31] S. Boyd and L. Vandenberghe, Convex optimization. Cambridge university press, 2004.

[32] T. Ferguson, A Course in Large Sample Theory: Texts in Statistical Science. Chapman \& Hall/CRC, 1996, vol. 38

[33] H. Ahmed, K. Jagannathan, and S. Bhashyam, "Queue-aware optimal resource allocation for the lte downlink - technical report," 2013. [Online]. Available: http://www.ee.iitm.ac.in/ krishnaj/globecomextended.pdf

[34] D. Pollard, A user's guide to measure theoretic probability. Cambridge University Press, 2001, vol. 8, p. 57. 